\title{
CONNECTED TRIPLE EXCITATIONS IN THE EQUATION-OF-MOTION COUPLED CLUSTER CALCULATIONS OF THE ENERGIES AND PROPERTIES OF EXCITED STATES: HCN MOLECULE*
}

\author{
MARTA WLOCH, MONIKA MUSIAL, STANISŁAW A.KUCHARSKI \\ Institute of Chemistry University of Silesia, Szkolna 9, 40-006 Katowice, Poland
}

(Rec. 1 December 2003)

\begin{abstract}
The recently developed equation-of-motion approach with full inclusion of the connected triple excitation [S. A. Kucharski, M. Włoch, M. Musiał, R. J. Bartlett, J. Chem. Phys., 115, 8263 (2001)] has been applied for the first time to calculate the adiabatic excitation energies. The two lowest excited states of the $\mathrm{HCN}$ molecule were studied and the equilibrium geometry and harmonic frequencies as well as the vertical excitation energies have been obtained and compared with the experimental data if the latter were available. The inclusion of the $T_{3}$ operators improves the results by 0.10 to $0.25 \mathrm{eV}$ depending on the basis set and the state considered.
\end{abstract}

\section{INTRODUCTION}

In the accurate quantum chemical calculations the coupled cluster (CC) method [1-11] plays a prominent role due to the fact that the convergence with the rank of the excitation operator is much faster than e.g. in the configuration interaction (CI) theory. With the inclusion of the $T_{4}[9]$ and $T_{5}[10,11]$ excitation operators one can achieve the accuracy of the order of tens of microhartree. We have to be aware that this is the accuracy of several orders lower than that reached with explicitly correlated Gaussians where the sub-microhartree is a standard level of accuracy [12-15]. The CC theory which has been primarily developed as a method for the calculation of the ground state properties, has recently gained anew momentum when used for the description of the excited states. The technique used for that purpose is based on the equation-of-motion approach introduced into the CC theory by the Gainesville group [16-21]. Equivalent, although formally different approach known as a linear response (CCLR) theory, which goes back to the works of Monkhorst [22], is being developed by the Aarhus group [23-25].

The CC scheme most commonly used in combination with the EOM approach is the CCSD model and its performance is well documented in literature [16-21]. This approach has been successfully used for the description of the excited states which are dominated by single excitations. Nowadays several efforts have been made to improve its accuracy also for the states with large double excitation character by developing CC schemes with inclusion of the higher-than-double excitation operators [26, 27]. This refers to the implementation of the approach with full inclusion of the connected triple excitations for the evaluation of the excited states energies [28]. The program developed in [28] is coded very efficiently both

\footnotetext{
* Dedicated to the memory of Professor Jacek Rychlewski
} 
at the CCSDT level (with quasilinear formulation of the CC equations) and at the CI step, where most of the terms engaging three- and four-body elements of the $\bar{H}$ operator were consequently factorized out.

In the EOM-CC approach an excited state is created by the action of an elementary excitation operator (5) on the CC reference state $\psi_{o}$ obtained within the CC formalism with an exponential $e^{T}$ operating on the Hartree-Fock reference, $\boldsymbol{\Phi}_{o}$. The expansion coefficients for the excited state are defined by the solution of the matrix eigenvalue equation obtained by the projection of the eigenvalue problem upon a suitable set of configurations. The diagonalization also yields directly the excitation energies. In other word, EOM-CC may be viewed as a diagonalization of the CC similarity transformed Hamiltonian, $\bar{H}$, in the CI configuration space.

The aim of the current work is to investigate the importance of the $T_{3}$ operator in the EOMCC evaluation of the equilibrium geometry and harmonic frequencies for the two lowest excited states of the HCN molecule. The analogous properties for the ground state were studied in [29], where also higher cluster operators, $T_{1}$ to $T_{5}$, were discussed. The results of [29] brought us to the conclusion that in order to calculate e.g. the equilibrium bond length with the high accuracy $(0.0001 \AA$ or better) one has to incorporate into the CC expansion the $T_{4}$ or even $T_{5}$ operators. On the other hand, the high accuracy calculations require using large basis sets and those two conditions are mutually prohibitive. The $\mathrm{CC}$ approaches including higher clusters $\left(\geq T_{3}\right.$ ) are computationally so demanding that the calculations are feasible only for small basis sets.

This apparent difficulty can be circumvented due to the observation made in papers $[30,31]$, where it was shown that for the certain classes of basis sets, like e.g. correlationconsistent basis sets of Dunning [32] the corrections due to the particular cluster operator are very stable and change very little when going from small to large basis sets. This observation, exploited in [30,31], made it possible to evaluate the equilibrium bond length and harmonic frequency with accuracy of the order of $0.0001 \mathrm{~A}$ and 1-2 $\mathrm{cm}^{-1}$, respectively. The procedure adopted there relies on the low rank CC calculations (CCSD or CCSD(T)) for large basis sets (e.g. cc-pV5Z or cc-pV6Z) and on the a posteriori corrections due to the higher cluster made on the basis of their evaluation for small basis sets (cc-pVDZ or cc-pVTZ). In the current work we use the cc-pVTZ and cc-pVQZ basis sets at the CCSD level - to assess the effect of the basis set size and for smaller basis sets, cc-pVDZ and PBS of Sadlej [33], we run full EOM-CCSDT calculations.

\section{THEORY}

Let us consider the Schrödinger equation

$$
H\left|\Psi_{k}\right\rangle=E_{k}\left|\Psi_{k}\right\rangle \quad k=1,2 \ldots
$$

assuming that $\Psi_{k}$ describes the $k$-th excited state. In the coupled-cluster formalism the reference wave function $\Psi_{o}$ is obtained by the action of the exponential $e^{T}$ 


$$
\left|\Psi_{o}\right\rangle=e^{T}\left|\Phi_{o}\right\rangle
$$

where $\left|\Phi_{o}\right\rangle$ is the reference determinant and $T$ is the cluster operator. Within the CCSDT model, the cluster operator is approximated as

$$
T=T_{1}+T_{2}+T_{3}
$$

and $T_{k}$ is defined as

$$
T_{k}=(k !)^{-2} \sum_{a b \ldots i j \ldots} \sum_{i j}^{a b} a^{\dagger} b^{\dagger} \ldots j i
$$

where the indices $a, b, \ldots(i, j, \ldots)$ refer to the virtual (occupied) one-particle levels. The cluster amplitudes, $t_{i j \ldots}^{a b \ldots}$, are obtained by solving the $\mathrm{CC}$ equations:

$$
\left\langle\Phi_{i j \ldots}^{a b \ldots}\left|\left(H e^{T}\right)_{c}\right| \Phi_{o}\right\rangle=0
$$

$\left\langle\begin{array}{l}a b \ldots \\ i j \ldots\end{array}\right|$ is a $k$-tuply excited determinant and the subscript $c$ in the above equation indicates that only connected diagrams should be considered.

Within the EOM formalism the $k$-state wave function $\left(\left|\Psi_{k}\right\rangle\right)$ is obtained by operating with the $R(k)$ operator on the ground state wave function $\left(\left|\Phi_{o}\right\rangle\right)$

$$
\left|\Psi_{k}\right\rangle=R(k)\left|\Psi_{o}\right\rangle
$$

The $R(k)$ is a linear (CI-like) excitation operator limited in this approach to the single, double and triple excitations:

$$
R(k)=r_{o}+R_{1}(k)+R_{2}(k)+R_{3}(k)
$$

or in the expanded form

$$
R(k)=r_{o}+\sum_{i a} r_{i}^{a}(k) a^{\dagger} i+\frac{1}{4} \sum_{a b} \sum_{i j} r_{i j}^{a b}(k) a^{\dagger} b^{\dagger} j i+\frac{1}{36} \sum_{a b c} \sum_{i j l} r_{i j l}^{a b c}(k) a^{\dagger} b^{\dagger} c^{\dagger} l j i
$$

Inserting the $\left|\Psi_{k}\right\rangle$ wave function, Eq. (6), into the Schrödinger equation, Eq. (1), we obtain - after simple algebra - the equation-of-motion

$$
\bar{H} \quad R(k)=\omega_{k} R(k)
$$

where $\bar{H}$ is the similarity transformed Hamiltonian, formally defined as

$$
\bar{H}=e^{-T} H e^{T}
$$

and $\omega_{k}$ is the energy change connected with the excitation process. The EOM-CCSDT requires solution of the CCSDT equations, Eq. (5), for $T_{1}, T_{2}$, and $T_{3}$ amplitudes and then 
the construction of the $\bar{H}$ operator, according to Eq. (10). To obtain the eigenvalues and eigenvectors we employ a direct diagonalization scheme [34] analogous to the Davidson method [35] for symmetric matrices. In case of the solution for the right-hand-side eigenvectors we require the results of the EOM-CC matrix right multiplying an arbitrary vector 5 ,i.e., $\bar{H} R$.

The form of $\bar{H}$ which is required for the construction and solution of the EOM-CCSDT equations in their standard form involves up to four-body elements. Emphasizing the manybody structure of $\bar{H}$, we may decompose it into individual $n$-body contributions, $I^{n}$, as follows:

$$
\bar{H}=I^{1}+I^{2}+I^{3}+I^{4}
$$

The many-body type of the $\bar{H}$ elements expressed through the $I_{t \ldots}^{r \ldots}$ amplitudes is determined by the number of indices i.e. $I_{r}^{s}, I_{t u}^{r s}, I_{u v w}^{r s t}$ and $I_{v w x y}^{r s t u}$ correspondto the $I^{1}, I^{2}, I^{3}$ and $I^{4}$ operators, respectively.

All elements of $\bar{H}$ are defined in the recursive way, see Ref. [9], i.e. the lower rank element can be used in the definition of the element of higher rank. The important thing is that once a given element is constructed it can be used in all equations.

The standard way of derivation of the EOM-CC equation assumes that we use in the equation all required $\bar{H}$ elements regardless of the complexity of the considered term. This means that in the standard version we employ all required three- and four-body terms.

Such a formulation of the EOM-CCSDT problem, although most natural one, would result in the high rank of the computational procedure both at the step of the $\bar{H}$ construction and at the step of the solution of the EOM equations. To avoid this, we need to apply a factorization scheme which would allow to eliminate the difficult terms i.e. those engaging the four-body $\bar{H}$ element and some of the three-body ones.

The factorization procedure, i.e. replacement of some $\bar{H}$ elements contracted with the $R$ operators with the appropriate intermediates contracted with the $T$ operator makes the evaluation of the $(\bar{H} R)$ quantities much more efficient. By the intermediate we understand here the quantity obtained by the contraction of the integral or $\bar{H}$ element with the $R$ operator. We should remember, however, that the $\bar{H}$ elements are computed only once in the whole process while the intermediates must be recomputed in each iteration. However, it does not change the general conclusion concerning this procedure.

\section{RESULTS}

The calculations carried out in the current work involve the optimization of the ground state geometry, optimization of the excited states geometry, calculation of the harmonic frequencies for both ground and excited states and evaluation of the vertical and adiabatic transition energies. Four different basis sets were used: correlation consistent basis set of Dunning [32], cc-pVDZ, cc-pVTZ and cc-pVQZ and the polarized basis set (PBS) of Sadlej 
[33]. Since the full inclusion of the connected triple excitations in the EOMCC formalism is a demanding computational scheme we adopted a following strategy in the calculations: for the smaller basis sets, i.e. cc-pVDZ and PBS we performed both the CCSD and CCSDT calculation, for the larger basis sets, i.e. cc-pVTZ and cc-pVQZ, only the EOMCCSD approach was used. The results for the smaller basis sets enabled us to estimate the $T_{3}$ effect for the studied property while the larger basis sets indicate the expected basis limit.

Tables 1 and 2 present the equilibrium geometry and harmonic frequencies for two small basis sets, cc-pVDZ and PBS, respectively. As it was mentioned before, the effect of theparticular cluster on the equilibrium geometry and on the harmonic frequency of the ground state has already been studied elsewhere [29]. Here it is confirmed that theinclusion of the $T_{3}$ operator increases the $\mathrm{HC}$ bond length by 0.0016 and $0.0018 \AA$ (for the cc-pVDZ and PBS basis sets, respectively) and by 0.0062 and $0.0064 \AA$ for the $\mathrm{CN}$ bond.

Table 1. Equilibrium geometry (in $\AA$ ) and harmonic frequencies (in $\mathrm{cm}^{-1}$ ) for ground and excited states of the HCN molecule (cc-pVDZ ${ }^{\text {a) }}$ basis set)

\begin{tabular}{lccccccccc}
\hline & \multicolumn{3}{c}{${ }^{1} \Sigma^{+}$} & \multicolumn{3}{c}{${ }^{1} A^{\prime \prime}$} & \multicolumn{3}{c}{${ }^{1} A^{\prime}$} \\
\cline { 2 - 10 } & CCSD & CCSDT & $\Delta^{\mathrm{b})}$ & CCSD & CCSDT & $\Delta^{\mathrm{b})}$ & CCSD & CCSDT & $\Delta^{\mathrm{b})}$ \\
\hline$R_{\mathrm{HC}}$ & 1.0810 & 1.0826 & 0.0016 & 1.1482 & 1.1506 & 0.0024 & 1.1889 & 1.2106 & 0.0217 \\
$R_{\mathrm{CN}}$ & 1.1688 & 1.1750 & 0.0062 & 1.3003 & 1.3131 & 0.0128 & 1.2989 & 1.3065 & 0.0076 \\
$\alpha_{\mathrm{HCN}}$ & $180^{\circ}$ & $180^{\circ}$ & $0^{\circ}$ & $121.0^{\circ}$ & $121.3^{\circ}$ & $0.3^{\circ}$ & $97.6^{\circ}$ & $95.4^{\circ}$ & $-2.2^{\circ}$ \\
$\omega_{1}$ & 3471 & 3447 & -24 & 2528 & 2482 & -46 & 2122 & 1874 & -248 \\
$\omega_{2}$ & 2157 & 2103 & -54 & 1571 & 1529 & -42 & 1353 & 1291 & -62 \\
$\omega_{3}$ & 732 & 708 & -24 & 918 & 956 & 38 & 650 & 483 & -167 \\
\hline
\end{tabular}

${ }^{\text {a) }}$ Ref. 32

b) $\Delta$ represents the difference between CCSDT and CCSD values

Table 2. Eqilibrium geometry (in $\AA$ ) and harmonic frequencies (in $\mathrm{cm}^{-1}$ ) for ground and excited states of the HCN molecule ( PBS $^{\text {a) }}$ basis set)

\begin{tabular}{|c|c|c|c|c|c|c|c|c|c|}
\hline & \multicolumn{3}{|c|}{${ }^{1} \Sigma^{+}$} & \multicolumn{3}{|c|}{ 'A ${ }^{\prime}$, } & \multicolumn{3}{|c|}{${ }^{1} A^{\prime}$} \\
\hline & CCSD & CCSDT & $\Delta^{\mathrm{b})}$ & CCSD & CCSDT & $\Delta^{\mathrm{b})}$ & CCSD & CCSDT & $\Delta^{\mathrm{b})}$ \\
\hline$R_{\mathrm{HC}}$ & 1.0831 & 1.0849 & 0.0018 & 1.1531 & 1.1565 & 0.0034 & 1.1996 & 1.2226 & 0.0230 \\
\hline$R_{\mathrm{CN}}$ & 1.1695 & 1.1759 & 0.0064 & 1.3026 & 1.3148 & 0.0122 & 1.2968 & 1.3034 & 0.0066 \\
\hline$\alpha_{\mathrm{HCN}}$ & $180^{\circ}$ & $180^{\circ}$ & $0^{\circ}$ & $120.8^{\circ}$ & $121.1^{\circ}$ & $0.3^{\circ}$ & $98.3^{\circ}$ & $96.7^{\circ}$ & $-1.6^{\circ}$ \\
\hline$\omega_{1}$ & 3404 & 3378 & -26 & 2511 & 2448 & -63 & 2067 & 1810 & -257 \\
\hline$\omega_{2}$ & 2129 & 2075 & -54 & 1545 & 1502 & -43 & 1347 & 1280 & -67 \\
\hline$\omega_{3}$ & 718 & 691 & -27 & 895 & 927 & 32 & 635 & 430 & -205 \\
\hline
\end{tabular}

a) Ref. 35

b) $\Delta$ represents the difference between CCSDT and CCSD values

The HCN system exists in the excited states as a nonlinear molecule and the computed bond lengths are significantly larger compared to the ground state values, e.g. HC bond is 
longer by $0.067 / 0.068 \AA$ for the ${ }^{1} A$ " state and by $0.108 / 0.129 \AA$ for the ${ }^{1} A$ ' state (CCSD/CCSDT). Similar behaviour is observed in the case of the $\mathrm{CN}$ bond. In both excited states the $\mathrm{CN}$ bond length increases to ca. $1.30 \AA$ compared to ca. $1.17 \AA$ for the ground state. The $\mathrm{HCN}$ angle for the ${ }^{1} A$ " state is close to $120^{\circ}$ and is significantly reduced, to ca. $95 / 98^{\circ}$, for the ${ }^{1} A^{\prime}$ state.

We observe also that for the excited states the $T_{3}$ effect is much more visible, confirming that the proper correlation treatment is more important for the excited states than for the ground one. The $T_{3}$ effect for the optimum bond lengths in the ${ }^{1} A$ " state is twice as large as the ground state $T_{3}$ effect. The next excited state is characterized by the substantial increase of the $\mathrm{HC}$ bond length and a very moderate change in the $\mathrm{CN}$ bond (comparable to the ground state effect). This indicates a certain weakening of the $\mathrm{HC}$ bond in the ${ }^{1} A$ ' state.

The computed harmonic frequencies show changes which are consistent with bond length effects. The stretching frequencies are significantly reduced in the excited states, e.g. the HC stretching frequency goes down from $3447 \mathrm{~cm}^{-1}$ for the ground state to $2482 \mathrm{~cm}^{-1}$ and 1874 for the ${ }^{1} A^{\prime \prime}$ and ${ }^{1} A^{\prime}$ states, respectively (both values refer to the CCSDT scheme). Similarly, theCN stretching frequency is reduced by 574 and $812 \mathrm{~cm}^{-1}$ for the ${ }^{1} A^{\prime \prime}$ and ${ }^{1} A^{\prime}$ states (CCSDT values), respectively. The $\omega_{3}$ frequency, representing the bending mode, behaves in a more irregular way: we observe its increase in the ${ }^{1} A$ " state by ca. $250 \mathrm{~cm}^{-1}$ and a substantial decrease for the ${ }^{1} A$ ' state. The $T_{3}$ effect for the harmonic frequencies is larger for the excited states but only for the $\mathrm{HC}$ stretching frequency. In particular the inclusion of the $T_{3}$ operator reduces the $\mathrm{HC}$ value for the ${ }^{1} A$ ' state by $248 \mathrm{~cm}^{-1}$. Also the bending frequency for the same state is more sensitive to the correlation treatment, the $T_{3}$ operator reduces the $\omega_{3}$ value by $167 \mathrm{~cm}^{-1}$.

In order to investigate the basis set effects we turn to Table 3, where we listed the CCSD results for the series of three correlation consistent basis sets: cc-pVDZ, cc-pVTZ and cc-pVQZ. In the column with the $\mathrm{pV} \infty \mathrm{Z}$ header we collected the values obtained by extrapolating the results to the complete basis set (CBS) limit [36]. The observed effects indicate - which is commonly known for the ground state - that by getting closer to the Hartree-Fock limit the bond lengths are reduced. We should be aware that the values collected in Table 3 refer to the CCSD method and realistically evaluating the bond lengths we should include the $T_{3}$ effect which works in the opposite direction, i.e. increases bond length. So e.g. the CBS limit for the $\mathrm{HC}$ bond for the ${ }^{1} A$ ' state is ca. $1.149 \AA$, see the last column of the Table 3. So taking into account the $T_{3}$ effect equal to ca. $0.022 \AA$ we can estimate the CBS limit of $\mathrm{HC}$ bond length in the ${ }^{1} A^{\prime}$ state equal to approximately $1.171 \AA$. Similar considerations the ${ }^{1} A^{\prime \prime}$ state give the value of $1.121 \AA$. The analogous procedure applied to the $\mathrm{CN}$ bond indicates the CBS limit to be equal to 1.283 and $1.266 \AA$ for the ${ }^{1} A^{\prime \prime}$ and ${ }^{1} A$ ' states, respectively. After inclusion of the estimated $T_{3}$ correction the final values are equal to 1.296 and $1.273 \AA$ for the two considered states, respectively. 
Table 3. Computed and extrapolated $\mathrm{d}^{\text {a) }}$ equilibrium geometry (in $\AA$ ) and harmonic frequencies (in $\mathrm{cm}^{-1}$ ) for ground and excited states of the HCN molecule at the CCSD level

\begin{tabular}{|c|c|c|c|c|c|c|c|c|c|c|c|c|c|c|}
\hline & \multicolumn{5}{|c|}{${ }^{1} \Sigma^{+}$} & \multicolumn{5}{|c|}{${ }^{\prime} A{ }^{\prime \prime}$} & \multicolumn{4}{|c|}{$\overline{{ }^{1} A^{\prime}}$} \\
\hline & $\mathrm{pVDZ}$ & pVTZ & $\mathrm{pVQZ}$ & $\mathrm{pV} \infty \mathrm{Z}$ & Exp. ${ }^{\text {b) }}$ & pVDZ & pVTZ & pVQZ & $\mathrm{pV} \infty \mathrm{Z}$ & Exp. $^{\text {c) }}$ & $\mathrm{pVDZ}$ & pVTZ & $\mathrm{pVQZ}$ & $\mathrm{pV} \infty \mathrm{Z}$ \\
\hline$R_{\mathrm{HC}}$ & 1.0810 & 1.0649 & 1.0648 & 1.0647 & 1.066 & 1.1482 & 1.1232 & 1.1208 & 1.1190 & 1.14 & 1.1889 & 1.1566 & 1.1521 & 1.1488 \\
\hline$R_{\mathrm{CN}}$ & 1.1688 & 1.1528 & 1.1491 & 1.1464 & 1.153 & 1.3003 & 1.2887 & 1.2851 & 1.2825 & 1.30 & 1.2989 & 1.2789 & 1.2717 & 1.2664 \\
\hline$\alpha_{\mathrm{HCN}}$ & $180^{\circ}$ & $180^{\circ}$ & $180^{\circ}$ & $180^{\circ}$ & $180^{\circ}$ & $121.0^{\circ}$ & $122.1^{\circ}$ & $122.4^{\circ}$ & $122.6^{\circ}$ & $125^{\circ}$ & $97.6^{\circ}$ & $103.0^{\circ}$ & $104.8^{\circ}$ & $106.1^{\circ}$ \\
\hline$\omega_{1}$ & 3471 & 3472 & 3475 & 3477 & 3440 & 2528 & 2646 & 2673 & 2693 & & 2122 & 2281 & 2320 & 2348 \\
\hline$\omega_{2}$ & 2157 & 2175 & 2200 & 2218 & 2128 & 1571 & 1578 & 1586 & 1592 & & 1353 & 1414 & 1442 & 1462 \\
\hline$\omega_{3}$ & 732 & 743 & 745 & 745 & 727 & 918 & 928 & 929 & 930 & & 650 & 460 & 430 & 408 \\
\hline
\end{tabular}

\footnotetext{
a) Ref. 36

b) bond length from Ref. 37, harmonic frequencies from Ref. 38

${ }^{c}$ bond length and angle from Ref. 39.
} 
The same reasoning can be applied to the harmonic frequencies. For the ${ }^{1} A$ " state the basis set effect is smaller, the CBS limit is equal to 2693, 1592 and $930 \mathrm{~cm}^{-1}$, for three considered modes. The ${ }^{1} A$ ' state is more sensitive to the size of the basis set: e.g. the HC stretching frequency increases by ca $200 \mathrm{~cm}^{-1}$ when going from double zeta to quadruple zeta basis set. Hence, in the realistic evaluation of the HC stretching frequency we should consider the extrapolated $2348 \mathrm{~cm}^{-1}$ value reduced by the $T_{3}$ effect $\left(-257 \mathrm{~cm}^{-1}-\right.$ PBS $)$ to give $2091 \mathrm{~cm}^{-1}$. The latter value, of course, can be further reduced by the inclusion into the calculations of the higher cluster operators.

Table 4. Vertical and adiabatic excitation energies (in eV) of the HCN molecule with EOM-CC methods

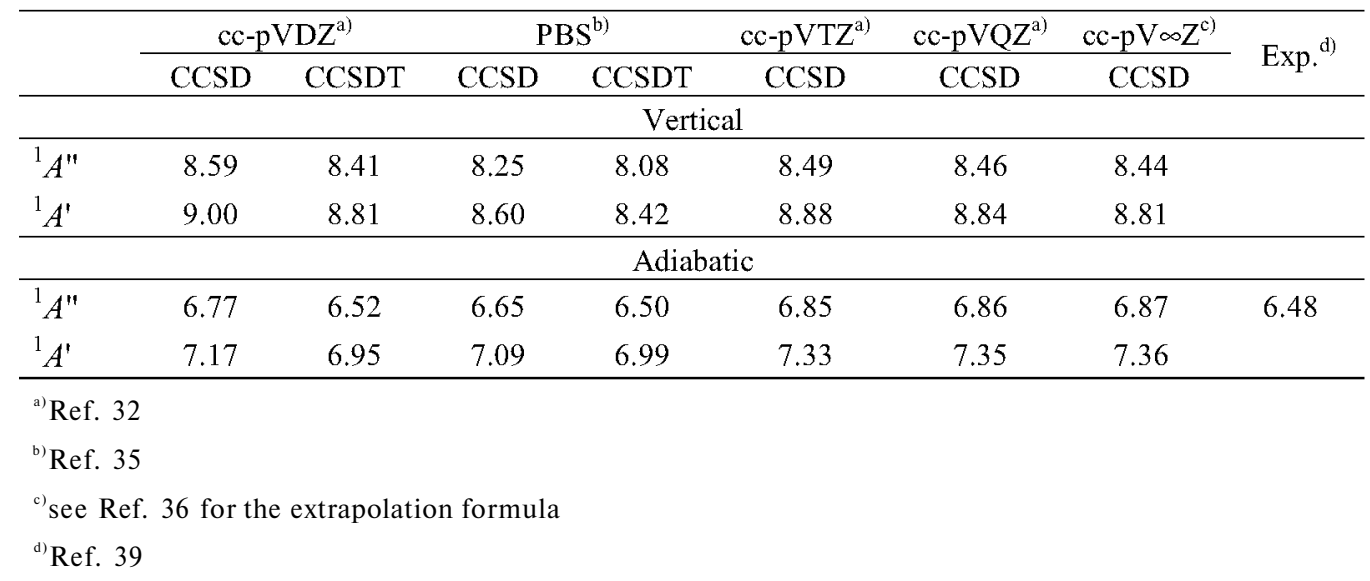

The excitation energy values are collected in Table 4. From the fact that the excited state geometry significantly differs from the ground state - see Tables 1-3 - it may be deduced that there occurs a considerable differentiation between vertical and adiabatic excitation energies. The data collected in Table 4 fully confirm this, e.g. the adiabatic excitation energy for the ${ }^{1} A$ " state is lower by ca. 1.6 to $1.9 \mathrm{eV}$ from its vertical counterpart. For the ${ }^{1} A$ ' state the decrease is similar i.e. between 1.4 and $1.9 \mathrm{eV}$. The $T_{3}$ effect is very stable for the vertical transitions, and is equal to $0.17-0.19 \mathrm{eV}$. The changes in the adiabatic values - due to the $T_{3}$ - are larger for the pVDZ basis set $(>0.2 \mathrm{eV})$ and distinctly smaller for the PBS basis set $\left(0.15 \mathrm{eV}\right.$ for ${ }^{1} A$ " and $0.10 \mathrm{eV}^{\text {for }}{ }^{1} A$ ).

Comparing the computed excitation energies for the sequence of the basis sets: pVDZ, pVTZ and pVQZ we observe rather a small effect connected with the basis set size, e.g. for the vertical values the ${ }^{1} \mathrm{~A}$ " state energy goes down from $8.59 \mathrm{eV}$ (pVDZ) to $8.46 \mathrm{eV}$ (pVQZ). Consequently, an extrapolation of the computed values to the CBS limit gives the value which is only slightly different from the cc-pVQZ results. The adiabatic values change in different direction: the excitation energy increases with the size of the basis set, but the net effect is even smaller than in the case of the vertical transitions. 


\section{CONCLUSIONS}

The EOM-CCSDT method, representing the state-of-the-art treatment of the excited states with coupled cluster theory, has been applied to study the excited states of the HCN molecule. The full inclusion of the $T_{3}$ operator provides much more reliable description of the equilibrium geometry, and - eo ipso - of the adiabatic excitation energies. The role of the $T_{3}$ operator is much more important in the description of certain excited states than in the case of the ground state. e.g. the $T_{3}$ effect on the $\mathrm{HC}$ bond length in the ${ }^{1} A^{\prime}$ state is $0.022 \AA$ compared to 0.002 for the ground state. The similar large $T_{3}$ effect is observed for the HC stretching frequency in the same state.

The excited states show also a slower convergence with the size of the basis sets, e.g. the extrapolated CBS value for the HC bond length is smaller by $0.004 \AA$ than that for the ccpVQ basis set, while for the ground state the same effect is equal to $0.0001 \AA$.

Since the experimental data are available only for the ${ }^{1} A^{\prime \prime}$ state the performance of the method can be assessed only with respect to this state. The PBS EOM-CCSDT adiabatic excitation energy, $6.50 \mathrm{eV}$, is in perfect agreement with the experimental value of $6.48 \mathrm{eV}$. The computed bond length values for the PBS basis set are slightly too large: 1.156 vs. $1.14 \AA$ and 1.315 vs. $1.30 \AA$. However, taking into account the basis set effect (i.e. CCSD pVœZ value corrected by the $T_{3}$ effect) we arrive at the 1.121 and $1.296 \AA$ values compared with the experimental 1.14 and $1.30 \AA$ results.

We realize that the EOM-CCSDT method, although significantly more accurate than e.g. the EOM-CCSD scheme, cannot be routinely applied to the large chemical systems. However, its usefulness and reliability in the description of the excited states of small molecules opens the important field of the possible applications, especially as these states are more difficult to access experimentally.

\section{Acknowledgments}

This work has been supported by the Committee for Scientific Research (KBN), Poland, under Grant No. 4 T09A01324.

\section{References}

[1] J. Cizek, J. Chem. Phys., 45, 4256 (1966); Advan. Chem. Phys., 14, 15 (1969); J. Paldus, J. Cizek and I. Shavitt, Phys. Rev., A5, 50 (1974).

[2] R. J. Bartlett, J. Phys. Chem., 93, 1697 (1989); R. J. Bartlett, in Modern Electronic Structure Theory, Part 1, ed. D.R. Yarkony, (World Scientific Publishing Co., New York, 1995), pp. 1047-1131; R. J. Bartlett and J. F. Stanton, in: Reviews in Computational Chemistry, Vol. 5, eds. K. B. Lipkowitz and D. B. Boyd (VCH Publishers, New York, 1994), pp. 65-169.

[3] G. D. Purvis III and R. J. Bartlett, J. Chem. Phys., 76, 1910 (1982).

[4] G. E. Scuseria, T. J. Lee, and H. F. Schaefer, Chem. Phys. Lett., 130, 236 (1986).

[5] Y. S. Lee, S. A. Kucharski and R. J. Bartlett, J. Chem. Phys., 81, 5906 (1984);

[6] J. Noga and R. J. Bartlett, J. Chem. Phys., 86, 7041 (1987); ibid. 89, 3401 (1988). 
[7] G. Scuseria and H. F. Schaefer III, Chem. Phys. Lett., 152, 382 (1988).

[8] J. D. Watts and R. J. Bartlett, J. Chem. Phys., 93, 6104 (1989).

[9] S. A. Kucharski and R. J. Bartlett, J. Chem. Phys., 97, 4282 (1992).

[10] M.Musiał, S. A. Kucharski and R. J. Bartlett, Chem. Phys. Lett., 320, 542 (2000).

[11] M. Musiał, S. A. Kucharski and R. J. Bartlett, J.Chem. Phys., 116, 4382 (2002).

[12] J. Komasa, J. Rychlewski, Chem. Phys. Lett., 320, 549 (2000).

[13] W. Cencek, J. Rychlewski, Chem. Phys. Lett., 342, 185 (2001).

[14] J. Komasa, J. Rychlewski, Mol. Phys., 91, 909 (1997).

[15] J. Rychlewski, Adv. Quantum Chem., 31, 173 (1999).

[16] J. F. Stanton and R. J. Bartlett, J. Chem. Phys., 98, 7029 (1993); J. Geertsen, M. Rittby, and R. J. Bartlett, Chem. Phys. Lett., 164, 57 (1989); D. C. Comeau and R. J. Bartlett, ibid. 207, 414 (1993).

[17] J. F. Stanton and J. Gauss, J. Chem. Phys., 100, 4695 (1994).

[18] J. D. Watts and R. J. Bartlett, Chem. Phys. Lett., 233, 81 (1995); 258, 581 (1996); J. Chem. Phys., 101, 3073 (1994); J. D. Watts and R. J. Bartlett, Spectrochim. Acta, Part A 55, 495 (1999).

[19] M. Nooijen and R. J. Bartlett, J. Chem. Phys., 106, 6441 (1997).

[20] L. Meissner and R. J. Bartlett, J. Chem. Phys., 94, 6670 (1991).

[21] L. Meissner and R. J. Bartlett, J. Chem. Phys., 102, 7490 (1995).

[22] H. Monkhorst, Int. J. Quantum Chem. Symp., 11, 421 (1977).

[23] H. Koch, H. J. Aa Jensen, P. Jørgensen, and T. Helgaker, J. Chem. Phys., 93, 3345 (1990).

[24] O. Christiansen, H. Koch, and P. Jørgensen, J. Chem. Phys., 105, 1451 (1996).

[25] O. Christiansen, H. Koch, P. Jørgensen, and J. Olsen, Chem. Phys. Lett., 256, 185 (1996).

[26] K. Kowalski and P. Piecuch, J. Chem. Phys., 113, 8490 (2000).

[27] K. Kowalski and P. Piecuch, J. Chem. Phys., 115, 643 (2001).

[28] S. A. Kucharski, M. Włoch, M. Musiał, and R. J. Bartlett, J. Chem. Phys., 115, 8263 (2001).

[29] M. Musiał, S. A. Kucharski, and R. J. Bartlett, J. Mol. Struct.(Theochem), 547, 269 (2001).

[30] S. A. Kucharski, J. D. Watts, and R. J. Bartlett, Chem. Phys. Lett., 302, 295 (1999).

[31] S. A. Kucharski, M. Kołaski, and R. J. Bartlett, J. Chem. Phys., 114, 692 (2001).

[32] T. H. Dunning, Jr., J. Chem. Phys., 90, 1007 (1989); R. A. Kendall, T. H. Dunning, Jr., and R. J. Harrison, ibid. 96, 6796 (1992); D. E. WoonandT. H. Dunning, Jr., ibid. 103, 4572 (1995).

[33] A. J. Sadlej, Collec. Czech. Chem. Commun., 53, 1995 (1988).

[34] K. Hirao and H. Nakatsuji, J. Comput. Phys., 45, 246 (1982).

[35] E. R. Davidson, J. Comput. Phys., 17, 87 (1975).

[36] A. Helgaker, T. Helgaker, P. Jorgensen, W. Klopper, H. Koch, J. Olsen, and A. K. Wilson, Chem. Phys. Lett. 286, 243 (1998).

[37] G. Winnewisser, A. G. Makai, and D. R. Johnson, J. Mol. Spectrosc., 39, 149 (1971).

[38] W. Quapp, J. Mol. Spectrosc., 125, 122 (1987).

[39] G. Herzberg, and K. K. Innes, Can. J. Phys., 35, 842 (1957). 